\title{
Correction to: Metric Properties of Semialgebraic Mappings
}

\author{
Krzysztof Kurdyka $^{1} \cdot$ Stanisław Spodzieja ${ }^{2}\left[\right.$ - Anna Szlachcińska ${ }^{2}$
}

Published online: 3 September 2019

(C) The Author(s) 2019

\section{Correction to: Discrete \& Computational Geometry (2016) 55:786-800 https://doi.org/10.1007/s00454-016-9776-4}

In some formulas in the original article we have omitted in the exponent the number of functions (or equivalently the dimension of the target) which is denoted by $m$. This shortcoming could lead to misunderstandings in applications.

1. In Corollary 2.2, inequality (2.6), the exponent $N+r-1$ should be replaced by $N+m+r-1$. Namely it should be

$$
\mathcal{L}_{0}^{\mathbb{R}}(F \mid X) \leq d(6 d-3)^{N+m+r-1}
$$

instead of

$$
\mathcal{L}_{0}^{\mathbb{R}}(F \mid X) \leq d(6 d-3)^{N+r-1} .
$$

Indeed, let $F: X \rightarrow \mathbb{R}^{m}$ be a continuous semialgebraic mapping, where $X \subset \mathbb{R}^{N}$ is a closed semialgebraic set. Assume that $0 \in X$ and $F(0)=0$. Set $r=r(X)+$ $r(\operatorname{graph} F)$ and $d=\max \{\kappa(X), \kappa(\operatorname{graph} F)\}$. Clearly

$$
\text { graph } F \subset \mathbb{R}^{N+m} \text {. }
$$

The original article can be found online at https://doi.org/10.1007/s00454-016-9776-4.

Krzysztof Kurdyka

Krzysztof.Kurdyka@univ-savoie.fr

Stanisław Spodzieja

spodziej@math.uni.lodz.pl

Anna Szlachcińska

anna_loch@wp.pl

1 Laboratoire de Mathematiques (LAMA), Universié Savoie Mont Blanc, UMR-5127 de CNRS, 73-376 Le Bourget-du-Lac Cedex, France

2 Faculty of Mathematics and Computer Science, University of Łódź, S. Banacha 22, 90-238 Łódź, Poland 
Therefore, it follows from (2.4) in Theorem 2.1 that

$|F(x)| \geq \operatorname{dist}((x, 0), \operatorname{graph} F) \geq C \operatorname{dist}\left((x, 0),\left[X \cap F^{-1}(0)\right] \times\{0\}\right)^{d(6 d-3)^{N+m+r-1}}$

for $(x, 0) \in U \cap[X \times\{0\}]$, where $U$ is a neighborhood $0 \in \mathbb{R}^{N+m}$. This gives the correct version of inequality (2.6).

2. Analogously, in Corollary 2.2, inequality (2.7), the exponent $N+r$ should be replaced by $N+m+r$. Namely it should be

$$
\mathcal{L}_{0}^{\mathbb{R}}(F \mid X) \leq \frac{(2 d-1)^{N+m+r}+1}{2}
$$

instead of

$$
\mathcal{L}_{0}^{\mathbb{R}}(F \mid X) \leq \frac{(2 d-1)^{N+r}+1}{2} .
$$

3. Since formula (2.6) was corrected, in the last paragraph of Remark 2.4 the statement "... with our estimate $\mathcal{L}_{0}^{\mathbb{R}}(F \mid X) \leq d(6 d-3)^{N+r-1}$..." should be replaced by "... with our estimate $\mathcal{L}_{0}^{\mathbb{R}}(F \mid X) \leq d(6 d-3)^{N+r+m-1} \ldots$...

4. In Corollary 3.3 (the first centered inequality), $m$ should be added in the exponent. Namely it should be

$$
|F(x)| \geq C\left(\frac{\operatorname{dist}\left(x, F^{-1}(0) \cap X\right)}{1+|x|^{d}}\right)^{d(6 d-3)^{N+m+r-1}} \quad \text { for } x \in X
$$

instead of

$$
|F(x)| \geq C\left(\frac{\operatorname{dist}\left(x, F^{-1}(0) \cap X\right)}{1+|x|^{d}}\right)^{d(6 d-3)^{N+r-1}} \quad \text { for } x \in X .
$$

5. Finally, in Corollary 3.3 (the second centered inequality), it should be

$$
\mathcal{L}_{\infty}^{\mathbb{R}}(F \mid X) \geq(1-d) d(6 d-3)^{N+m+r-1}
$$

instead of

$$
\mathcal{L}_{\infty}^{\mathbb{R}}(F \mid X) \geq(1-d) d(6 d-3)^{N+r-1} .
$$

These corrections do not affect the asymptotics of the Łojasiewicz exponent.

6. The correct number of the grant from the Polish National Science Centre is 2012/07/B/ST1/03293.

Publisher's Note Springer Nature remains neutral with regard to jurisdictional claims in published maps and institutional affiliations. 